\title{
Interactive versus passive dynamics and the exploratory analysis of multivariate data
}

\author{
FRANK M. MARCHAK and LORI CASE MARCHAK \\ TASC, Reading, Massachusetts
}

\begin{abstract}
In two experiments, we examined subjects' ability to infer structure from three-dimensional data presented dynamically in interactive versus passive mode and in static scatterplots. Accuracy of estimating the number of clusters in three-dimensional data sets was measured for each of these conditions, as a function of cluster discriminability.
\end{abstract}

The use of graphical methods in exploratory data analysis has been advocated by a variety of investigators (e.g., Tufte, 1990; Tukey, 1977). A plethora of personalcomputer programs (e.g., SYSTAT, MacSpin, and Spyglass) have been developed which allow the user to graphically manipulate the form and presentation of data sets. However, the literature provides little guidance in the selection of the most effective and accurate graphical methods needed to reveal the structure in a data set or how different representational approaches, such as static plots versus dynamic rotation, affect data interpretability.

Toward this end, Marchak and Whitney (1990) compared the dynamic depiction of three-dimensional data with traditional scatterplots, particularly with respect to the ability of observers to extract cluster information from multidimensional data sets. They presented subjects with nine data sets that differed in the number of clusters present (one, two, or three) and the distances between clusters (near, middle, or far). The data were presented as three two-dimensional static scatterplots, dynamically rotated $360^{\circ}$ about each of three axes and animated according to a fourth variable. The subject's task was to determine if there were one, two, or three data clusters in each set.

Surprisingly, the findings showed no significant difference between static and dynamic presentation techniques. It was noted that one possible explanation of this outcome was that the experimental conditions constrained the subject's ability to interact with the data. In a real analysis situation, one would use rotation and animation jointly, choosing different views for animation and generally examining the data more thoroughly.

In the present experiments, we explore this possibility in detail. Our first experiment replicates the original work of Marchak and Whitney (1990), using a within-subjects design. In Marchak and Whitney's study, 24 subjects each viewed nine data sets, which were presented both on a

The authors wish to thank David A. Whitney for assistance in the generation of the stimuli. Correspondence should be addressed to Frank M. Marchak, TASC, 55 Walkers Brook Drive, Reading, MA 01867. computer screen and on paper printouts. Our replication uses 5 subjects, each viewing 180 cluster sets, with both dynamic and static cluster sets presented on a computer screen. This method controls the presentation media and allows a more thorough examination of any effects by lending itself to more rigorous statistical analysis.

In our second experiment, we examine the effects of interactivity on data interpretation. Using dynamic rotation, the subjects participate in two conditions. In the interactive condition, they are given $15 \mathrm{sec}$ to explore each data set using the rotation controls to view the data across $360^{\circ}$ in any manner they choose. In the passive condition, the data are rotated by the experimenter in each of the three dimensions for $5 \mathrm{sec}$. If interactivity allows etter interpretations, the subjects should be more accurate in determining the number of clusters present in the interactive condition.

\section{EXPERIMENT 1}

\section{Method}

Subjects. Five engineers from TASC, all with normal or corrected-to-normal vision, served as volunteers.

Design. A $2 \times 3 \times 3$ within-subjects design was used: two graphic presentation types (dynamic rotation and static), three cluster differences (near, middle, and far), and three different numbers of clusters present (one, two, and three). Presentation order of graphic types was balanced across subjects; cluster distances and cluster numbers were randomly distributed across trials.

Stimuli. One hundred eighty three-dimensional cluster groupings were generated. All were composed of 90 points sampled from a normal distribution with specified $3 \times 3$ correlation matrices with standard deviations of 5 . In the three-group cluster set, 20 groups consisting of 30 points sampled from the above distribution were generated. Twenty two-group and 20 one-group cluster sets, each made up of 45 and 90 points, respectively, were also sampled from the same distribution.

The mean for each cluster of points was variable and adjusted to change the distance between the clusters. Using the standard euclidean metric to measure distance, the 
intercluster distances were 3,5 , and 7 for the near, middle, and far conditions, respectively (Figure 1).

Apparatus. The stimuli were displayed on a Macintosh II computer. For the dynamic condition, the stimuli were presented using MacSpin (Figure 2), a dynamic graphical data-analysis package produced by D2 Software (see Donoho, Donoho, \& Gasko, 1988). The stimuli were rotated about each of the three spatial axes using the rotation functions provided by the software.

In the static condition, three views of each point set were presented simultaneously in the $x-y, y-z$, and $x-z$ dimensions using PLUS, a hypermedia presentation program (Figure 3). Each stimulus set was presented sequentially for all 180 cluster sets.

Procedure. The 180 data sets were entered into MacSpin and were printed as three two-dimensional scatterplots on individual cards in PLUS. In the dynamic condition, each data set was rotated $360^{\circ}$ about each of the three axes. In the static condition, three scatterplots of the $x-y$, $y-z$, and $x-z$ dimensions were presented simultaneously.

The subjects viewed each data set and were asked to determine whether there were one, two, or three data clusters in each set. The order of conditions and the order of data-set presentation were varied across subjects. Responses were recorded on a response sheet, with no time limit imposed on responding.

\section{Results and Discussion}

The total number of errors across presentation method showed no significant difference, with a mean number of errors of $\mathbf{1 4 . 9}$ for the dynamic condition and $\mathbf{1 8 . 1}$ for the static condition. Collapsing the data across number of clusters, repeated measures analyses of variance were performed on cluster difference and presentation method. There was no significant difference for presentation method, whereas for cluster distance, $F(2,8)=20.93$, $p<.001$, with means of $26.5,7.4$, and 15.5 for near, middle, and far conditions. Figure 4 shows the interaction plot of presentation method and cluster distance. The subjects tended to make twice as many errors in the neardistance condition as in the far-distance condition, with the least number of errors in the middle-distance condition, independent of presentation method.

These findings replicate the original findings of Marchak and Whitney (1990), showing no significant benefit in total number correct for dynamic presentation of scatterplots versus static presentation techniques. This implies that the addition of dynamics alone does not necessarily facilitate data interpretation. As mentioned in the original study, this effect may be due to the constrained nature of the task that prevents the subjects from actively interacting with the data sets. In Experiment 2, this possibility was explicitly examined.

\section{EXPERIMENT 2}

\section{Method}

Subjects. Five engineers from TASC, all with normal or corrected-to-normal vision, served as volunteers. None of the subjects in Experiment 2 had participated in Experiment 1 .

Design. A $2 \times 3 \times 3$ within-subjects design was used: two graphic presentation techniques (interactive and passive), three cluster differences (near, middle, and far),

\section{NUMBER OF CLUSTERS}

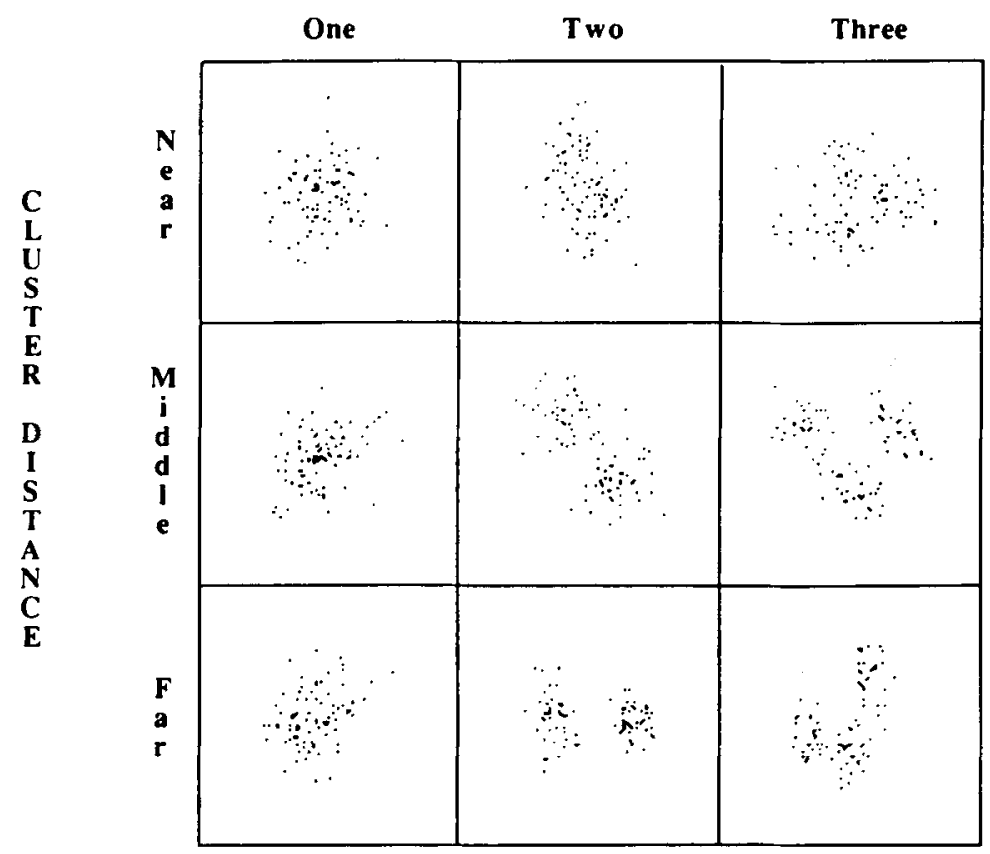

Figure 1. Representative cluster sets. 


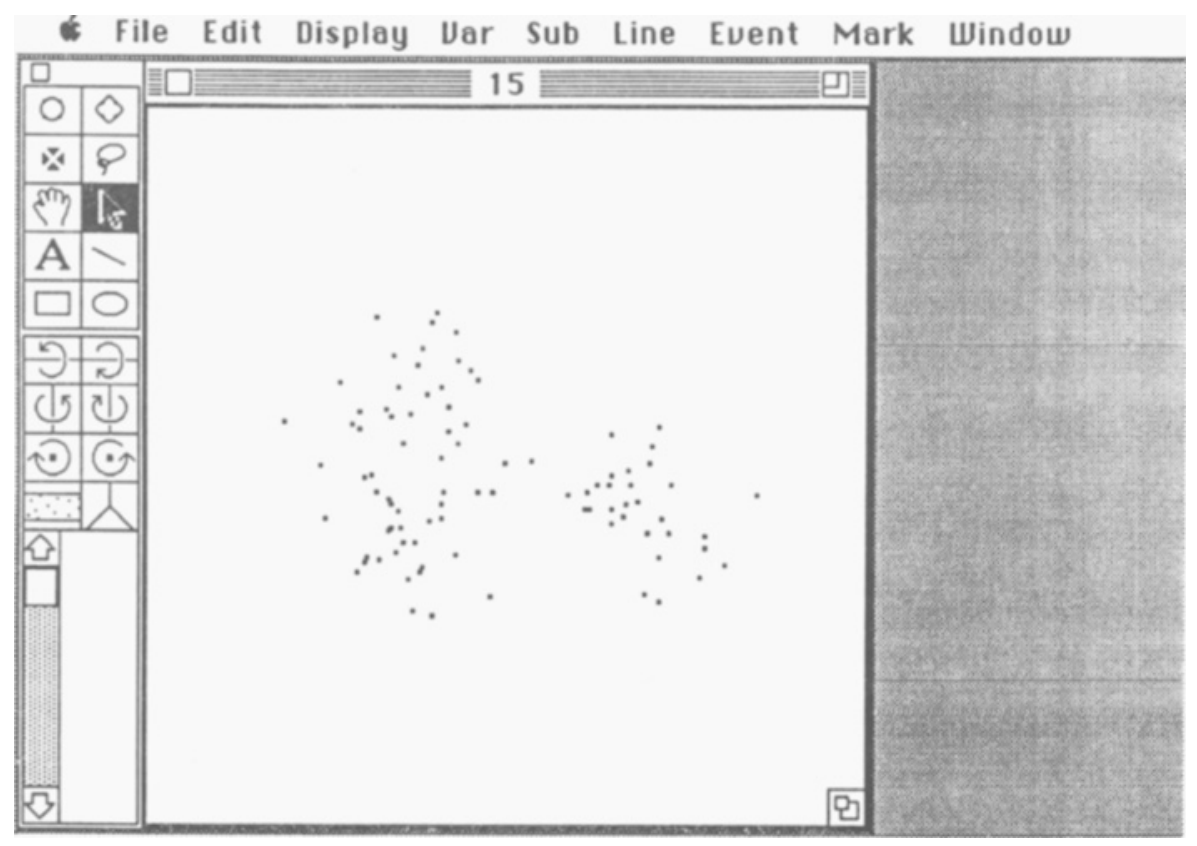

Figure 2. Sample MacSpin data presentation.

and three numbers of clusters (one, two, and three). Order of presentation techniques was balanced across subjects; cluster distances and cluster numbers were randomly distributed across trials.

Stimuli and Apparatus. One hundred eighty threedimensional cluster groupings were generated, in the same manner as in Experiment 1. Half of the stimuli were used in the passive condition and half in the interactive condition.
The stimuli were displayed on a Macintosh II computer using MacSpin, in the same manner as in the dynamic condition of Experiment 1.

Procedure. The 180 data sets were entered into MacSpin as described in Experiment 1. In the interactive condition, the subjects were given $15 \mathrm{sec}$ to use the rotation controls of MacSpin to examine each data set in whatever manner they chose. In the passive condition, each data set was rotated $360^{\circ}$ for $5 \mathrm{sec}$ about each of the three axes.

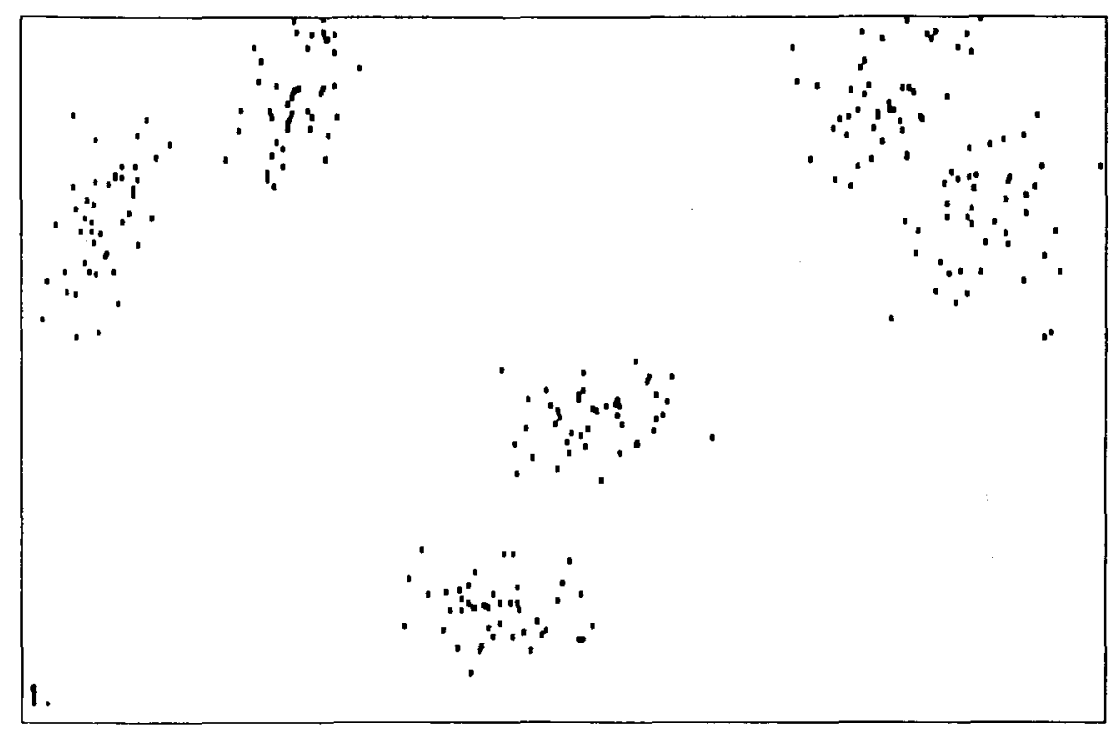

Figure 3. Sample static cluster representation. 


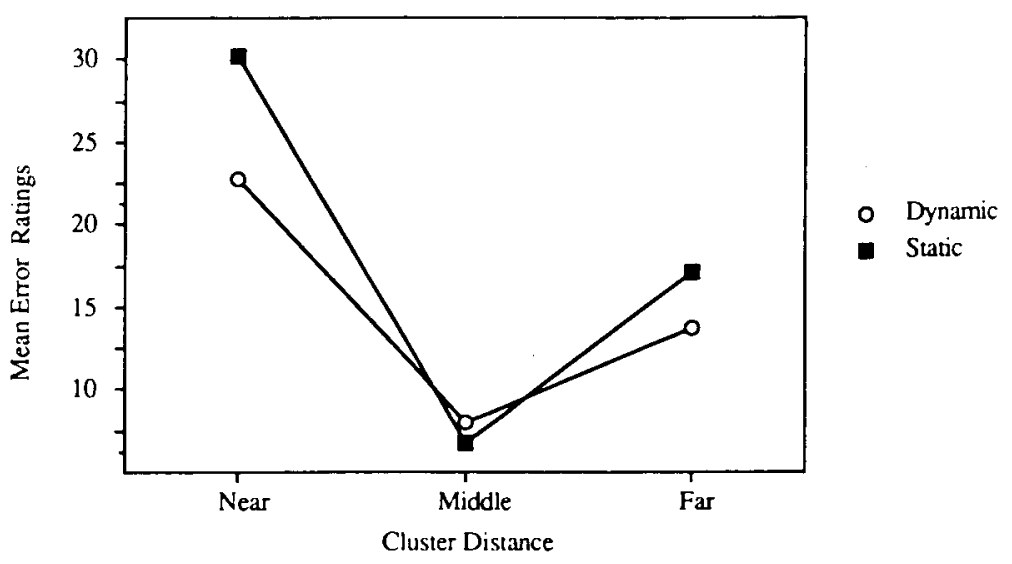

Figure 4. Interaction of presentation method and cluster distance.

In both conditions, the subjects viewed each data set and determined the number of clusters present. Responses were recorded on a response sheet with no time limitation.

\section{Results and Discussion}

The total number of errors across interaction method showed no significant difference, with a mean number of errors of 7.1 for the interactive condition and 7.1 for the passive condition. Collapsing the data across number of clusters, repeated measures analyses of variance were performed on cluster difference and interaction method. There was no significant difference for interaction method, whereas for cluster distance, $F(2,8)=13.59$, $p=.002$, with mean number of errors of $12.5,2.3$, and 6.5 for near, middle, and far conditions. Figure 5 shows the plot of interaction method and cluster distance. The subjects tended to make twice as many errors in the neardistance condition as in the far-distance condition, with the least number of errors in the middle-distance condition, independent of interaction method.

These findings suggest that lack of interaction with the data sets has no significant effect on the correct interpretation of the number of clusters in these data sets. The subjects made essentially the same number of errors regardless of the level of interaction.

\section{GENERAL DISCUSSION}

In the present experiments, we attempted to replicate the original findings of Marchak and Whitney (1990), in which no significant benefit of dynamic versus static data depiction was found. Furthermore, we explored the effect of interactivity on dynamic presentation methods. Experiment 1 differed from the original study, having fewer subjects view a far larger number of data sets. Nonetheless, no difference was found between the presentation methods

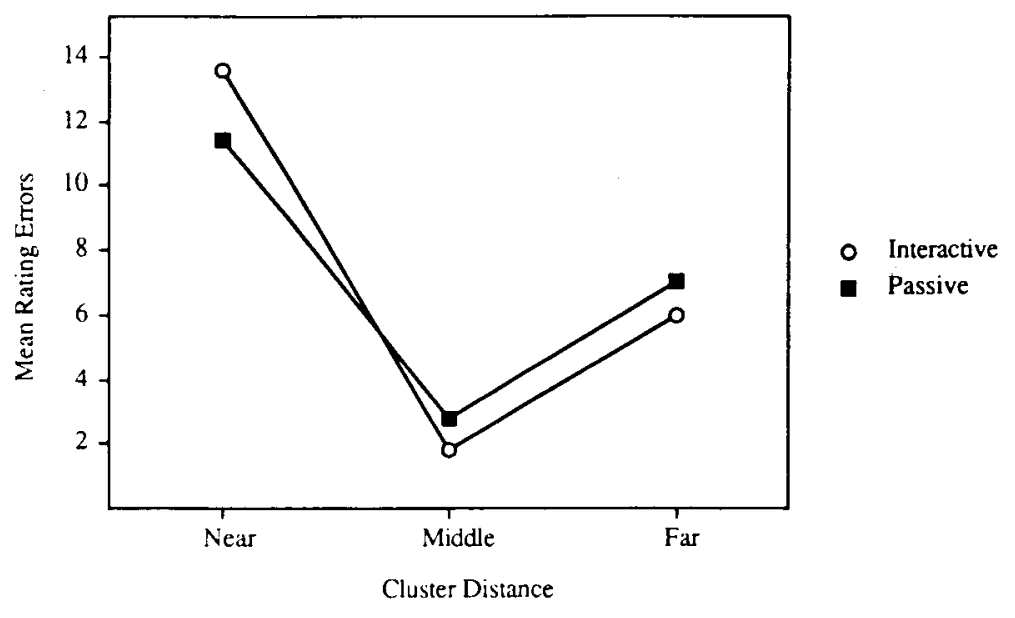

Figure 5. Interaction of passive versus interactive methods and cluster distance. 
on ability to determine cluster numbers, similar to Marchak and Whitney (1990). In Experiment 2, we examined the effect of interactivity on dynamic data interpretation, but we found no significant benefit of interaction over passive observation.

Together, these findings suggest that, at least for data sets consisting of point clouds, there is little or no benefit to be realized from adding interactive dynamic capabilities to data interpretation. This is a surprising finding, given the level of effort currently devoted to interactive graphical techniques (e.g., McCormick, DeFanti, \& Brown, 1987). One possible explanation could be that determining the number of clusters present does not effectively take advantage of any benefits provided by the addition of dynamical graphics. In many instances, such as the interpretation of sonar data, the task is to identify some structure from a noisy background environment. In this case, the addition of animation leads to structure emerging from the correlated events; such structure is not evident in a static representation. Further examination in- volving determination of structure in noisy backgrounds, with such stimuli as those used by Uttal (1983), may provide better evidence for the benefits of interactive graphical techniques.

\section{REFERENCES}

Donoho, A. W., Donoho, D. L., \& Gasko, M. (1988). MacSpin: Dynamic graphics on a desktop computer. In W. S. Cleveland \& M. E. McGill (Eds.), Dynamic graphics for statistics (pp. 331-351). Monterey, CA: Wadsworth.

MarchaK, F. M., \& Whitney, D. A. (1990). Dynamic graphics in the exploratory analysis of multivariate data. Behavior Research Methods, Instruments, \& Computers, 22, 176-178.

McCormick, B. H., DeFAnT, T. A., \& Brown, M. D. (EDs.). (1987). Visualization in scientific computing [Special issue]. Computer Graphics, 21.

TuFte, E. R. (1990). Envisioning information. Cheshire, CT: Graphic Press.

TUKEY, J. W. (1977). Exploratory data analysis. Reading, MA: Addison-Wesley.

UTTAL, W. R. (1983). Visual form detection in 3-dimensional space. Hillsdale, NJ: Erlbaum. 